\title{
FATORES ASSOCIADOS AO ABANDONO DO TRATAMENTO DA TUBERCULOSE: UMA REVISÃO INTEGRATIVA DA LITERATURA
}

\section{FACTORS ASSOCIATED WITH ABANDONMENT OF TUBERCULOSIS TREATMENT: AN INTEGRATIVE REVIEW OF THE LITERATURE}

Nytale Lindsay Cardoso Portela ${ }^{1}$

Resumo: A saúde pública, atualmente, enfrenta um grande desafio: reduzir as taxas de abandono do tratamento da tuberculose. O conhecimento dos fatores associados ao abandono torna-se, portanto, necessário para mudar essa realidade. Este estudo teve como objetivo descrever, a partir de evidências disponíveis na literatura, os principais fatores associados ao abandono do tratamento da tuberculose. Trata-se de uma revisão integrativa da literatura de 14 artigos publicados no período de 2005 a 2014, nas bases de dados LILACS e SciELO. Como resultado, constatou-se que os fatores associados ao abandono do tratamento da tuberculose podem estar relacionados ao paciente e ao estilo de vida, aos serviços de saúde e à terapêutica. Conclui-se que este estudo é de suma importância para a população, profissionais e gestores, visto que permite o conhecimento dos fatores relacionados ao abandono do tratamento e, por conseguinte, o planejamento de estratégias para diminuir as taxas de abandono.

Palavras-chave: adesão à medicação; recusa do paciente ao tratamento; cooperação do paciente; pacientes desistentes do tratamento; tuberculose.

Abstract: Public health currently faces a major challenge: reducing the dropout rates for tuberculosis treatment. Therefore, knowledge of the factors associated with dropout becomes necessary to change this reality. This study aimed to describe, from evidence available in the literature, the main factors associated with abandonment of tuberculosis treatment. This is an integrative review of 14 articles published between 2005-2014 in the LILACS and SCIELO databases. As a result, it was found that the factors associated with noncompliance with treatment for tuberculosis could be related to the patient and their lifestyle, as well as health services and therapy. In conclusion, this study is of paramount importance to the people, professionals, and managers, as it provides information about the factors related to the abandonment of treatment and, therefore, planning of strategies to reduce dropout rates.

Keywords: medication adherence; treatment refusal; patient compliance; patient dropouts; tuberculosis.

\footnotetext{
${ }^{1}$ Especialista em Enfermagem do Trabalho e Saúde Pública e Saúde da Família - Faculdade de Ensino Superior Dom Bosco - FacDomBosco, Brasil e graduada em Enfermagem - Universidade Estadual do Maranhão - UEMA, Brasil. Email: nytalelindsay@hotmail.com.
} 


\section{INTRODUÇÃO}

A tuberculose (TB) é uma doença infectocontagiosa, cujo agente etiológico é o Mycobacterium tuberculosis, também conhecido como bacilo de Koch. Sua propagação se dá por meio de gotículas contendo os bacilos expelidos por um doente com TB pulmonar ao tossir, espirrar ou falar em voz alta. Quando pessoas sadias inalam essas gotículas, ocorre a infecção tuberculosa e pode haver o desenvolvimento da doença (BRASIL, 2002a).

Denomina-se caso de TB, todo indivíduo com diagnóstico confirmado por baciloscopia ou cultura e aquele em que o médico, com base nos dados clínico-epidemiológicos e no resultado de exames complementares, firma o diagnóstico de TB (BRASIL, 2002b).

No Brasil, no ano de 2012, a taxa de incidência de TB foi de 37,28 casos por 100.000 habitantes, sendo mais incidente nos estados do Rio de Janeiro (70,57 casos por 100.000 habitantes), Amazonas (67,64 casos por 100.000 habitantes) e Pernambuco (52,21 casos por 100.000 habitantes) (BRASIL, 2012).

Um dos principais desafios para o controle da tuberculose, atualmente, é reduzir as taxas de abandono do tratamento, pois isso implica na persistência da fonte de infecção, no aumento das taxas de mortalidade e de recidivas, além de facilitar o desenvolvimento de cepas de bacilos resistentes (GOIS, 2009).

Conceitualmente, o abandono do tratamento ocorre quando o paciente deixa de ir à Unidade Básica de Saúde por mais de 30 dias consecutivos, após a data agendada para seu retorno. Quando o tratamento é supervisionado, é considerado abandono quando o doente deixa de tomar a medicação por 30 dias a partir da última tomada da droga (BRASIL, 2002b).

No Brasil, a taxa de abandono de tratamento é alta, situando-se em 17\%. Isso deve-se a diversos fatores, tais como: estigma da tuberculose, analfabetismo, a não aceitação da doença, o fato de considerar-se curado antes da cura efetiva, o não apoio dos familiares no tratamento, impossibilidade de comparecer às consultas no horário do agendamento, intolerância medicamentosa, alcoolismo, dentre outros (RIBEIRO et al., 2000).

Dessa forma, o objetivo deste estudo é descrever, a partir de evidências disponíveis na literatura, os principais fatores associados ao abandono do tratamento da tuberculose.

\section{METODOLOGIA}

Trata-se de uma revisão integrativa da literatura, que compreende a análise de pesquisa que possibilita a síntese do conhecimento de um determinado assunto, além de mostrar as lacunas que precisam ser preenchidas por meio da realização de novos estudos (POLIT; BECK, 2005).

Para a elaboração da presente revisão integrativa, foram seguidos os procedimentos metodológicos: formulação da questão e dos objetivos da revisão; estabelecimento de 
critérios para seleção dos artigos; categorização dos estudos; avaliação dos estudos incluídos na revisão integrativa; análise dos dados e apresentação dos resultados (WHITEMORE; KNAFL, 2005).

A questão norteadora do presente estudo foi: quais os fatores associados ao abandono do tratamento da tuberculose?

A seleção do material foi feita nos meses de dezembro de 2014 e janeiro de 2015. Para a seleção dos artigos, foram utilizadas duas bases de dados eletrônicas: LILACS (Literatura Latino Americana e do Caribe em Ciências Sociais e da Saúde) e SciELO (Scientific Electronic Library Online). Os descritores foram selecionados a partir da terminologia em saúde consultada no DeCS (Descritores em Ciências da Saúde) da Biblioteca Virtual em Saúde; são eles: adesão à medicação; recusa do paciente ao tratamento; cooperação do paciente; pacientes desistentes do tratamento; estes, por sua vez, foram combinados com o descritor tuberculose.

Os critérios de inclusão definidos foram: artigos originais, disponíveis na íntegra, publicados em português, inglês ou espanhol, no período compreendido de 2005 a 2014. Os critérios de exclusão foram: textos incompletos; artigos que não abordaram diretamente o tema do presente estudo ou que não foram realizados no Brasil; artigos de revisão; e repetição de um mesmo artigo na mesma base de dados ou em diferentes bases de dados, neste caso, considerou-se apenas um deles.

A partir dos descritores utilizados, obteve-se um total de 174 publicações na base de dados LILACS. Mediante os critérios de inclusão, selecionaram-se 96 artigos, porém houve repetição de 27 artigos e 05 eram artigos de revisão, resultando em um total de 64 artigos desta base de dados. Na base de dados SciELO, foram encontrados 11 artigos a partir dos descritores. Destes, 10 foram selecionados mediante os critérios de inclusão, no entanto, houve repetição de 03 artigos e 01 era artigo de revisão, resultando em 06 artigos.

Dos 70 artigos obtidos (64 da LILACS e 06 da SciELO), 06 foram excluídos por haver repetição entre as bases de dados e, após a leitura dos resumos, constatou-se que 50 não abordavam o tema deste estudo ou não foram estudos realizados no Brasil, resultando em 14 artigos para leitura na íntegra.

Após a leitura aprofundada dos artigos, elaboraram-se três quadros ilustrando os resultados obtidos, identificando título do artigo, idioma, periódico, ano de publicação, tipo de estudo e fatores associados ao abandono do tratamento da tuberculose.

\section{RESULTADOS E DISCUSSÃO}

A partir da análise dos 14 artigos, estabeleceram-se algumas variáveis para apreciação das produções científicas, conforme se observa no Quadro 1. Os artigos selecionados foram identificados por ordem alfabética como A1 a A14. 
No que diz respeito ao idioma, constata-se no Quadro 1 que, dos 14 artigos científicos relacionados ao tema, 13 estavam em português e um em inglês.

\section{Quadro 1 - Distribuição dos artigos selecionados, segundo título e idioma. Caxias- MA, Brasil, 2015}

\begin{tabular}{|c|c|c|}
\hline A & Título & Idioma \\
\hline $\bar{A} 1$ & $\begin{array}{l}\text { Abandono anunciado ao tratamento da tuberculose em uma Unidade de } \\
\text { Saúde da Família do Recife - A perspectiva do usuário }\end{array}$ & Português \\
\hline $\mathrm{A} 2$ & $\begin{array}{l}\text { Abandono do tratamento da tuberculose e integralidade da atenção na } \\
\text { Estratégia Saúde da Família }\end{array}$ & Português \\
\hline A3 & Abandono do tratamento de tuberculose em co-infectados TB/HIV & Português \\
\hline A4 & $\begin{array}{l}\text { Factors associated with treatment failure, dropout, and death in a cohort } \\
\text { of tuberculosis patients in Recife, Pernambuco State, Brazil }\end{array}$ & Inglês \\
\hline A5 & $\begin{array}{l}\text { Fatores associados ao abandono do tratamento da tuberculose pulmonar } \\
\text { no Maranhão, Brasil, no período de } 2001 \text { a } 2010\end{array}$ & Português \\
\hline $\mathrm{A} 6$ & $\begin{array}{l}\text { Fatores associados ao abandono do tratamento de tuberculose em } \\
\text { indivíduos acompanhados em unidades de saúde de referência na cidade } \\
\text { do Recife, Estado de Pernambuco, Brasil, entre } 2005 \text { e } 2010\end{array}$ & Português \\
\hline A7 & $\begin{array}{l}\text { Fatores associados ao abandono do tratamento de tuberculose no estado } \\
\text { do Paraná }\end{array}$ & Po \\
\hline A8 & $\begin{array}{l}\text { Fatores associados ao abandono do tratamento da tuberculose nos } \\
\text { serviços de atenção básica em dois municípios brasileiros, Manaus e } \\
\text { Fortaleza, } 2006 \text { a } 2008\end{array}$ & Português \\
\hline A9 & $\begin{array}{l}\text { Fatores determinantes para o abandono do tratamento da tuberculose: } \\
\text { representações dos usuários de um hospital público }\end{array}$ & Português \\
\hline A10 & $\begin{array}{l}\text { Fatores preditores para o abandono do tratamento da tuberculose } \\
\text { pulmonar preconizado pelo Ministério da Saúde do Brasil na cidade de } \\
\text { Porto Alegre (RS) }\end{array}$ & Português \\
\hline A11 & $\begin{array}{l}\text { Pacientes vivendo com HIV/AIDS e coinfecção tuberculose: dificuldades } \\
\text { associadas à adesão ou abandono do tratamento }\end{array}$ & Português \\
\hline A12 & $\begin{array}{l}\text { Perfil de casos de tuberculose notificados e fatores associados ao } \\
\text { abandono, Belo Horizonte, MG }\end{array}$ & Português \\
\hline A13 & $\begin{array}{l}\text { Prevalência de abandono do tratamento da tuberculose e fatores } \\
\text { associados no município de Sapucaia do Sul (RS), Brasil, 2000-200 }\end{array}$ & Português \\
\hline A14 & $\begin{array}{l}\text { Tratamento da tuberculose em unidades de saúde da família: histórias de } \\
\text { abandono }\end{array}$ & Português \\
\hline
\end{tabular}

Legenda: A - Artigo.

Em relação ao periódico, observa-se, no Quadro 2, que dois artigos foram publicados na Revista Texto \& Contexto Enfermagem e dois nos Cadernos de Saúde Pública. Os outros periódicos tiveram apenas uma publicação acerca do tema deste estudo, são eles: Revista de APS, Revista da Escola de Enfermagem da USP, Epidemiologia e Serviços de Saúde, Acta Paulista de Enfermagem, Cadernos Saúde Coletiva, Saúde em Debate, Jornal Brasileiro de Pneumologia, Revista Gaúcha de Enfermagem, Revista de Saúde Pública e Revista Brasileira de Epidemiologia.

Quanto ao ano de publicação, cinco artigos foram publicados em 2012, três em 2007, dois em 2011, dois em 2014, um em 2010 e um em 2013, demonstrando que dentro do recorte temporal selecionado (2005-2014), houve lacunas de publicações, não havendo 
artigos nos anos de 2005, 2006, 2008 e 2009 (Quadro 2).

A respeito do tipo de estudo, predominou os de natureza qualitativa (05 artigos), seguidos dos estudos de caso-controle (04), transversal (02), quali-quantitativo (01), de coorte (01) e ecológico (01), como pode-se observar no Quadro 2.

\section{Quadro 2 - Distribuição dos artigos selecionados, segundo o periódico, o ano de publicação e o tipo de estudo. Caxias-MA, Brasil, 2015}

\begin{tabular}{|c|l|l|l|}
\hline A & \multicolumn{1}{|c|}{ Periódico } & Ano & \multicolumn{1}{c|}{ Tipo de estudo } \\
\hline A1 & Rev. APS & 2012 & Estudo quali-quantitativo \\
\hline A2 & Texto Contexto Enferm. & 2012 & Estudo qualitativo \\
\hline A3 & Rev Esc Enferm USP & 2010 & Estudo qualitativo \\
\hline A4 & Cad. Saúde Pública & 2007 & Estudo de coorte \\
\hline A5 & Cad. Saúde Pública & 2014 & Estudo transversal analítico \\
\hline A6 & Epidemiol. Serv. Saúde & 2013 & Estudo de caso-controle \\
\hline A7 & Acta Paul Enferm. & 2012 & Estudo do tipo ecológico \\
\hline A8 & Cad. Saúde Colet. & 2012 & Estudo de caso-controle \\
\hline A9 & Saúde Debate & 2014 & Estudo exploratório, descritivo e qualitativo \\
\hline A10 & J Bras Pneumol. & 2011 & Estudo de caso-controle \\
\hline A11 & Rev Gaúcha Enferm. & 2012 & Estudo qualitativo \\
\hline A12 & Rev Saúde Pública & 2007 & Estudo de caso-controle \\
\hline A13 & Rev Bras Epidemiol & 2011 & Estudo transversal \\
\hline A14 & Texto Contexto Enferm. & 2007 & Estudo qualitativo \\
\hline
\end{tabular}

Legenda: A - Artigo.

Quanto aos resultados dos estudos, encontraram-se os fatores associados ao abandono do tratamento da tuberculose, conforme o Quadro 3. Percebe-se que os fatores associados ao abandono do tratamento da tuberculose podem estar relacionados ao paciente e ao estilo de vida, aos serviços de saúde e à terapêutica.

Quadro 3 - Fatores associados ao abandono do tratamento da tuberculose. CaxiasMA, Brasil, 2015

\begin{tabular}{|c|l|}
\hline A & \multicolumn{1}{|c|}{ Fatores associados ao abandono do tratamento da tuberculose } \\
\hline A1 & $\begin{array}{l}\text { O contexto social, a dependência do álcool, os efeitos dos fármacos, a organização do } \\
\text { processo de trabalho do serviço de saúde e a carência de conhecimentos acerca da } \\
\text { doença e do tratamento. }\end{array}$ \\
\hline A2 & $\begin{array}{l}\text { O modo como os profissionais da Estratégia Saúde da Família (ESF) têm } \\
\text { desempenhado o cuidado ao doente de tuberculose (TB) e sua família, o processo de } \\
\text { organização dos serviços de saúde, a falta de informação dos usuários sobre a } \\
\text { doença o o frágil acompanhamento em outros serviços de atenção à saúde, para além } \\
\text { da ESF. }\end{array}$ \\
\hline A3 & $\begin{array}{l}\text { A baixa condição socioeconômica, os efeitos adversos dos medicamentos, o uso de } \\
\text { drogas lícitas, as questões relacionadas à estrutura física, organização do processo de } \\
\text { trabalho para o controle da TB e as dificuldades de acesso ao serviço de saúde. Além } \\
\text { disso, a coinfecção TB/HIV favorece o abandono, pois há maior dificuldade para }\end{array}$ \\
\hline
\end{tabular}




\begin{tabular}{|l|l|}
\hline & $\begin{array}{l}\text { controlar esses casos seja por aspectos relativos ao próprio tratamento, seja pela falta } \\
\text { de motivação trazida pelo sentimento de iminência de morte. }\end{array}$ \\
\hline A4 & Grupo etário, tratamento prévio para tuberculose e analfabetismo. \\
\hline A5 & $\begin{array}{l}\text { Faixa etária de } 20-39 \text { anos, escolaridade (1-8 anos de estudo), analfabetismo, } \\
\text { alcoolismo e doença mental. }\end{array}$ \\
\hline A6 & Baixa escolaridade e histórico de abandono prévio. \\
\hline A7 & $\begin{array}{l}\text { Idade jovem, etilismo, desemprego, baixa escolaridade e reingresso após abandono } \\
\text { do tratamento. }\end{array}$ \\
\hline A8 & Sexo masculino, baixa classe econômica, tabagismo, etilismo, uso de drogas ilícitas. \\
\hline A9 & $\begin{array}{l}\text { Etilismo, uso de drogas, reações adversas à medicação, problemas socioeconômicos, } \\
\text { motivos religiosos, dificuldades de acesso ao tratamento, deficiência do vínculo com } \\
\text { os profissionais de saúde envolvidos no tratamento e a sensação de cura antes do } \\
\text { término do tratamento. }\end{array}$ \\
\hline A10 & $\begin{array}{l}\text { Etilismo (com ou sem a concomitância de uso de drogas ilícitas), infecção por HIV, fato } \\
\text { de o paciente que não residir com familiares e baixo nível de escolaridade. }\end{array}$ \\
\hline A11 & $\begin{array}{l}\text { Consumo de drogas ilícitas e uso do álcool. } \\
\text { A12 }\end{array}$ Uso de drogas e de bebidas alcoólicas e a melhora clínica. \\
\hline A13 & $\begin{array}{l}\text { Sexo masculino, indivíduos que reingressaram no Programa e pessoas que } \\
\text { apresentavam AlDS. O abandono foi mais frequente nas faixas etárias de } 30 \text { a } 39 \\
\text { anos e de } 20 \text { a } 29 \text { anos. }\end{array}$ \\
\hline A14 & $\begin{array}{l}\text { O desconhecimento e as crenças do doente acerca da tuberculose, dos hábitos } \\
\text { prejudiciais à saúde, com destaque para o etilismo como doença associada, e de } \\
\text { problemas econômicos e sociais, como o desemprego; a ocorrência de efeitos } \\
\text { colaterais; a melhora dos sintomas, criando a ilusão da cura antes da conclusão do } \\
\text { tratamento; o longo tempo de tratamento e a grande quantidade de comprimidos que } \\
\text { devem ser ingeridos. }\end{array}$ \\
\hline
\end{tabular}

Legenda: A - Artigo.

\subsection{Fatores relacionados ao paciente e ao estilo de vida}

Os fatores relacionados ao paciente e ao estilo de vida correlacionam-se, principalmente, com o seu perfil socioeconômico e cultural e com a experiência anterior de tratamento (SOUSA FILHO, 2012).

Estudos relataram que o etilismo é um dos principais fatores relacionados ao abandono do tratamento da tuberculose, sendo encontrado em 10 dos 14 estudos analisados. O consumo de bebida alcoólica deve servir de alerta para o profissional de saúde, que deve prestar uma assistência diferenciada, pois o etilismo torna o indivíduo mais vulnerável a abandonar o tratamento, além de agravar o quadro clínico, elevando a possibilidade de progressão para doença cavitária crônica (OLIVEIRA; ANTUNES, 2012; SÁ et al., 2007).

O tabagismo apareceu como significativo somente no estudo do tipo caso-controle, realizado por Braga et al. (2012) com 434 indivíduos (92 casos e 342 controles), assistidos pelos serviços de atenção básica de Manaus e Fortaleza, estando presente em $56,5 \%$, entre os casos, e em $31,9 \%$, entre os controles.

Além das drogas lícitas, as drogas ilícitas também foram citadas como fatores associados ao abandono, em quatro estudos. O uso de drogas, como o crack, é prejudicial à saúde do homem e ao bom funcionamento do indivíduo, especialmente, quando este já está acometido por alguma enfermidade. Além disso, o uso de drogas lícitas ou ilícitas associado 
ao tratamento, aumenta a chance de intolerância, podendo levar ao abandono (COUTO et al., 2014).

Outros fatores encontrados foram os socioeconômicos, como a baixa condição socioeconômica e o baixo nível de escolaridade, que segundo o estudo de Rodrigues et al. (2010), estão diretamente relacionados, levando à interrupção do tratamento. Além desses, Furlan, Oliveira e Marcon (2012) e Sá et al. (2007) citam o desemprego como fator associado ao abandono do tratamento da tuberculose. Neste último caso, isto deve-se à priorização da subsistência, que leva ao adiamento da procura para atendimento médico (PAIXÃO; GONTIJO, 2007).

A situação socioeconômica está diretamente ligada ao abandono do tratamento, pois os grupos com baixo nível socioeconômico são mais vulneráveis a adoecer por TB, sendo necessário, portanto, que a equipe de saúde propicie um melhor planejamento do tratamento, evitando que o paciente o abandone devido, por exemplo, à falta de meios para arcar com a condução em caso do tratamento supervisionado (LOPES, 2010).

O desconhecimento e as crenças dos usuários acerca da doença e do tratamento também foram mencionados como fatores relacionados ao abandono do tratamento tuberculostático. Oliveira e Antunes (2012) afirmam que quanto menos se tem conhecimento sobre a tuberculose, maiores são os riscos de não concluir o esquema terapêutico, sendo necessário o desenvolvimento de uma orientação contínua e singularizada ao longo de todo tratamento.

A falta de ações de educação em saúde sobre TB, de acordo com Alves et al. (2012), faz com que o usuário não assuma uma atitude motivacional e transformadora na busca pela mudança de seu estado de saúde, sendo importante, portanto, que as equipes de saúde incluam ações educativas para o doente e sua família, que não se restrinjam a palestras e panfletos.

O histórico de abandono prévio é outro fator que merece destaque. Silva, Andrade e Cardoso (2013), em estudo realizado com 711 pacientes, constataram que os indivíduos com histórico de abandono prévio apresentaram 7,2 vezes mais chance de abandonar o tratamento quando comparados aos casos novos. Ademais, Furlan, Oliveira e Marcon (2012) ressaltam que há risco de o paciente adquirir resistência às drogas, o que torna o tratamento mais caro, desencadeando maior risco à população em geral.

Alguns estudos relataram, ainda, que idade jovem, principalmente indivíduos entre 2029 anos; sexo masculino; infecção por HIV/AIDS; presença de doença mental; motivos religiosos; e o fato de o paciente não residir com familiares também podem estar relacionados ao abandono do tratamento da tuberculose (ALBUQUERQUE et al., 2007; BRAGA et al., 2012; CAMPANI; MOREIRA; TIETBOHEL, 2011; COUTO et al., 2014; FURLAN; OLIVEIRA; MARCON, 2012; HECK; COSTA; NUNES, 2011; RODRIGUES et al., 2010; SILVA; MOURA; CALDAS, 2014). 


\subsection{Fatores relacionados aos serviços de saúde}

Quanto aos motivos relacionados aos serviços de saúde, tem-se: a organização do processo de trabalho do serviço de saúde, o modo como os profissionais da Estratégia Saúde da Família tem desempenhado o cuidado ao doente e sua família e a deficiência do vínculo com os profissionais de saúde.

O serviço de saúde tem importante papel no controle da doença. Diante disso, a própria organização ou o mau funcionamento do serviço pode vir a dificultar as vantagens obtidas em um diagnóstico precoce e repercutir na motivação do doente, interferindo no tratamento e na sua cura. A demora no atendimento e os horários incompatíveis fazem com que os pacientes deixem de procurar os serviços e até a abandonar o tratamento, devido ao medo de perder o emprego por causa de faltas e atrasos (OLIVEIRA; ANTUNES, 2012).

O modo como o profissional de saúde assiste o paciente também é um fator importante. Alves et al. (2012) afirmam que o cuidado é limitado à verificação da ingesta ou não do medicamento e a alguns aspectos clínicos, não havendo, portanto, uma assistência pautada na integralidade. Mencionam, ainda, que ausência da escuta pelo profissional durante a consulta leva ao distanciamento nas relações entre profissional e usuário, comprometendo no processo terapêutico.

A escolha do profissional de referência, para Oliveira e Antunes (2012), é essencial para o sucesso terapêutico, pois facilita a negociação das ações propostas com o usuário e consequente gerência do cuidado. Para isso, é necessário que o profissional desenvolva habilidade de comunicação, com o intuito de minimizar o abandono relacionado à inadequada assimilação das informações e permitir a relação profissional-paciente de modo a promover a interação terapêutica.

Outros fatores relatados foram: o frágil acompanhamento em outros serviços de atenção à saúde, para além da ESF, as questões relacionadas à estrutura física, as dificuldades de acesso ao serviço de saúde e as dificuldades de acesso ao tratamento.

\subsection{Fatores relacionados à terapêutica}

Com relação aos fatores preditivos de abandono do tratamento da tuberculose ligados à terapêutica, identificaram-se os efeitos adversos dos fármacos, a melhora clínica (sensação de cura antes do término do tratamento), o longo tempo de tratamento e a grande quantidade de comprimidos que devem ser ingeridos.

Os efeitos adversos dos fármacos podem ser náusea, vômito, dor abdominal, escurecimento da urina, tontura, dentre outros. O abandono do tratamento devido essas reações indesejáveis indicam a falta de orientação do usuário pelo profissional de saúde, sendo essencial que o profissional informe os aspectos básicos do tratamento ao paciente, de forma a reduzir o índice de abandono. Ademais, é necessário que os profissionais monitorem os clientes quanto ao surgimento das reações adversas e oriente-os com 
medidas apropriadas, conforme o caso (OLIVEIRA; ANTUNES, 2012).

A melhora clínica que ocorre no início do tratamento, principalmente no final do primeiro mês e início do segundo, faz com que o cliente pense que já está curado, parando, assim, com a terapêutica antituberculosa (COUTO et al., 2014; SÁ et al., 2007). No estudo de Paixão e Gontijo (2007), este foi o segundo fator mais relatado pelos pacientes, o que mostra a necessidade do profissional de saúde informar ao paciente que o início do tratamento leva à assintomatologia e ao bom estado geral e, que mesmo havendo essa melhora, é de suma importância que o tratamento seja realizado por completo.

O tempo de duração do tratamento e a quantidade de medicamentos interferem na adesão, pois os esquemas terapêuticos são complexos para os pacientes, exigindo um grande empenho deles, que precisam adaptar sua alimentação, horários e ritmo diário para cumprir o tratamento. Ademais, ingerir muitos comprimidos de uma única vez causa medo, pois o doente teme os efeitos desagradáveis (GOIS, 2009).

\section{CONCLUSÃO}

O presente estudo permitiu identificar os principais fatores associados ao abandono do tratamento, tais como: uso de drogas lícitas e ilícitas, baixas condições socioeconômicas, organização do processo de trabalho do serviço de saúde, deficiência do vínculo com os profissionais de saúde, efeitos adversos dos fármacos, melhora clínica, longo tempo de tratamento e a grande quantidade de comprimidos que devem ser ingeridos, dentre outros.

Entretanto, não se pode determinar que os fatores citados são os únicos que determinam o abandono da terapêutica, visto que os fatores relacionados ao paciente, ao estilo de vida, aos serviços de saúde e à terapêutica podem ser distintos em locais que não foram estudados nos artigos selecionados.

Diante disso, há a necessidade de serem realizados outros estudos acerca da temática, buscando identificar outros possíveis fatores, visto que o conhecimento desses fatores é importante para que profissionais de saúde e gestores planejem estratégias para diminuir as taxas de abandono e, consequentemente, reduzir a incidência da doença, as taxas de mortalidade e a resistência aos fármacos.

\section{REFERÊNCIAS}

ALBUQUERQUE, M. F. P. M. et al. Factors associated with treatment failure, dropout, and death in a cohort of tuberculosis patients in Recife, Pernambuco State, Brazil. Cad. saúde pública, Rio de Janeiro, v. 23, n. 7, p. 1573-1582, 2007.

ALVES, R. S. et al. Abandono do tratamento da tuberculose e integralidade da atenção na Estratégia Saúde da Família. Texto \& contexto enferm., Florianópolis, v. 21, n. 3, p. 650657, 2012. 
BRAGA, J. U. et al. Fatores associados ao abandono do tratamento da tuberculose nos serviços de atenção básica em dois municípios brasileiros, Manaus e Fortaleza, 2006 a 2008. Cad. Saúde Coletiva, Rio de Janeiro, v. 20, n. 2, p. 225-233, 2012.

BRASIL. Ministério da Saúde. Manual técnico para o controle da tuberculose: cadernos de atenção básica. 6. ed. rev. e atual. Brasília: Ministério da Saúde, 2002a.

Tuberculose: guia de vigilância epidemiológica. Brasília: Ministério da Saúde, Fundação Nacional de Saúde, 2002b.

BRASIL. Ministério da Saúde. Secretaria de Vigilância em Saúde. IDB - INDICADORES E DADOS BÁSICOS. Rede Interagencial de Informações para a Saúde (RIPSA). D.2.2 Taxa de incidência de tuberculose, 2012. Disponível em: <http://tabnet.datasus.gov.br/cgi/tabcgi.exe?idb2012/d0202.def>. Acesso em: 21 de janeiro de 2015.

CAMPANI, S. T. A.; MOREIRA, J. S.; TIETBOHEL, C. N. Fatores preditores para o abandono do tratamento da tuberculose pulmonar preconizado pelo Ministério da Saúde do Brasil na cidade de Porto Alegre (RS). J. bras. pneumol., Brasília, v. 37, n. 6, p. 776-782, 2011.

COUTO, D. S. et al. Fatores determinantes para o abandono do tratamento da tuberculose: representações dos usuários de um hospital público. Saúde Debate, Londrina, v. 38, n. 102, p. 572-581, 2014.

FURLAN, M. C. R.; OLIVEIRA, S. P.; MARCON, S. S. Fatores associados ao abandono do tratamento de tuberculose no estado do Paraná. Acta paul. enferm., São Paulo, v. 25, n. esp. 1, p. 108-114, 2012.

GOIS, G. A. S. Abandono ao tratamento: desafio para o controle da tuberculose. 2009. 108p. Dissertação (Mestrado em Enfermagem) - Universidade Federal da Paraíba, João Pessoa (PB), 2009.

HECK, M. A.; COSTA, J. S. D.; NUNES, M. F. Prevalência de abandono do tratamento da tuberculose e fatores associados no município de Sapucaia do Sul (RS), Brasil, 2000-2008. Rev. bras. epidemiol., São Paulo, v. 14, n. 3, p. 478-485, 2011.

LOPES, A. O. Tuberculose um problema de Saúde Pública: causas do abandono do tratamento. 2010. 63p. Monografia (Graduação em Enfermagem) - Cento Universitário São Camilo, São Paulo, 2010.

OLIVEIRA, J. F.; ANTUNES, M. B. C. Abandono anunciado ao tratamento da tuberculose em uma Unidade de Saúde da Família do Recife: a perspectiva do usuário. Rev. APS, Juiz de Fora v. 15, n. 1, p. 4-13, 2012.

PAIXÃO, L. M. M.; GONTIJO, E. D. Perfil de casos de tuberculose notificados e fatores associados ao abandono, Belo Horizonte, MG. Rev. Saúde Pública, São Paulo, v. 41, n. 2, p. 205-213, 2007. 
POLIT, D. F.; BECK, C. T. Using research in evidence-based nursing practice. In: POLIT, D.F.; BECK, C.T. (eds.). Essentials of nursing research. Methods, appraisal and utilization. Philadelphia (USA): Lippincott Williams \& Wilkins, 2005. p. 457-94.

RIBEIRO, S. A. et al. Estudo caso-controle de indicadores de abandono em doentes com tuberculose. J. Pneumol., São Paulo, v. 26, n. 6, p. 291-296, 2000.

RODRIGUES, I. L. A. et al. Abandono do tratamento de tuberculose em co-infectados TB/HIV. Rev. Esc. Enferm. USP., São Paulo, v. 44, n. 2, p. 383-387, 2010.

SÁ, L. D. et al. Tratamento da tuberculose em Unidades de Saúde da Família: histórias de abandono. Texto \& contexto enferm., Florianópolis, v. 16, n. 4, p. 712-718, 2007.

SILVA, C. C. A. V.; ANDRADE, M. S.; CARDOSO, M. D. Fatores associados ao abandono do tratamento de tuberculose em indivíduos acompanhados em unidades de saúde de referência na cidade de Recife, Estado de Pernambuco, Brasil, entre 2005 e 2010. Epidemiol. serv. saúde., Brasília, v. 22, n. 1, p. 77-85, 2013.

SILVA, P. F.; MOURA, G. S.; CALDAS, A. J. M. Fatores associados ao abandono do tratamento da tuberculose pulmonar no Maranhão, Brasil, no período de 2001 a 2010. Cad. Saúde Pública, Rio de Janeiro, v. 30, n. 8, p. 1745-1754, 2014.

SOUSA FILHO, M. P. et al. Pacientes vivendo com HIV/AIDS e coinfecção tuberculose: dificuldades associadas à adesão ou ao abandono do tratamento. Rev. gaúch. enferm., Porto Alegre, v. 33, n. 2, p. 139-145, 2012.

WHITEMORE, R.; KNAFL, K. The integrative review: update methodology. J. adv. nurs., Oxford, v. 52, n. 5, p. 546-553, 2005. 